\title{
Video Article \\ Differential Effects of Lipid-lowering Drugs in Modulating Morphology of Cholesterol Particles
}

\author{
Shanmugavel Madasamy ${ }^{1}$, David Liu ${ }^{1}$, Jason Lundry ${ }^{1}$, Benjamin Alderete ${ }^{2}$, Raymond Kong ${ }^{2}$, J. Paul Robinson ${ }^{3}$, Alan H.B. Wu ${ }^{1,4}$, Edward \\ P. Amento 5 \\ ${ }^{1}$ Plaxgen Inc \\ ${ }^{2}$ Millipore Sigma \\ ${ }^{3}$ Cytometry Laboratories, Purdue University \\ ${ }^{4}$ San Francisco General Hospital, 2M16 Clinical Chemistry, University of California, San Francisco \\ ${ }^{5}$ Molecular Medicine Research Institute
}

Correspondence to: Shanmugavel Madasamy at shan@plaxgen.com

URL: https://www.jove.com/video/56596

DOI: doi:10.3791/56596

Keywords: Medicine, Issue 129, Cholesterol particles morphology, lipid-lowering drugs, plaque array, atherosclerosis, imaging flow cytometry, cardiovascular diagnosis

Date Published: $11 / 10 / 2017$

Citation: Madasamy, S., Liu, D., Lundry, J., Alderete, B., Kong, R., Robinson, J.P., Wu, A.H., Amento, E.P. Differential Effects of Lipid-lowering Drugs in Modulating Morphology of Cholesterol Particles. J. Vis. Exp. (129), e56596, doi:10.3791/56596 (2017).

\section{Abstract}

Treatment of dyslipidemia patients with lipid-lowering drugs leads to a significant reduction in low-density lipoproteins (LDL) level and a low to moderate level of increase in high-density lipoprotein (HDL) cholesterol in plasma. However, a possible role of these drugs in altering morphology and distribution of cholesterol particles is poorly understood. Here, we describe the in vitro evaluation of lipid-lowering drug effects in modulating morphological features of cholesterol particles using the plaque array method in combination with imaging flow cytometry. Image analyses of the cholesterol particles indicated that lovastatin, simvastatin, ezetimibe, and atorvastatin induce the formation of both globular and linear strand-shaped particles, whereas niacin, fibrates, fluvastatin, and rosuvastatin induce the formation of only globular-shaped particles. Next, purified very low-density lipoprotein (VLDL) and LDL particles incubated with these drugs showed changes in the morphology and image texture of cholesterol particles subpopulations. Furthermore, screening of 50 serum samples revealed the presence of a higher level of linear shaped HDL cholesterol particles in subjects with dyslipidemia (mean of $18.3 \%$ ) compared to the age-matched normal (mean of $11.1 \%$ ) samples. We also observed considerable variations in lipid-lowering drug effects on reducing linear shaped LDL and HDL cholesterol particles formation in serum samples. These findings indicate that lipid-lowering drugs, in addition to their cell-mediated hypolipidemic effects, may directly modulate morphology of cholesterol particles by a non-enzymatic mechanism of action. The outcomes of these results have potential to inform diagnosis of atherosclerosis and predict optimal lipid-lowering therapy.

\section{Video Link}

The video component of this article can be found at https://www.jove.com/video/56596/

Introduction

Numerous clinical studies have demonstrated beneficial effects of the lipid-lowering drugs in reducing plasma levels of low-density lipoproteins (LDL) cholesterol and a low to moderate level of increase in high-density lipoprotein (HDL) cholesterol, which prevents both primary and secondary incidences of atherosclerosis-related adverse cardiovascular events ${ }^{1,2,3,4,5}$. Statins, a group of HMG-CoA reductase enzyme inhibitors, block endogenous cholesterol synthesis in the liver that in turn lead to lower circulating levels of LDL cholesterol in the blood ${ }^{6,7}$. Likewise, the lipid lowering effect of niacin is mediated by its direct and noncompetitive inhibition of hepatocyte diacylglycerol acyltransferase-2, a key liver enzyme involved in triglyceride synthesis ${ }^{8}$. Comparatively, ezetimibe reduces plasma level of LDL by limiting absorption of exogenous

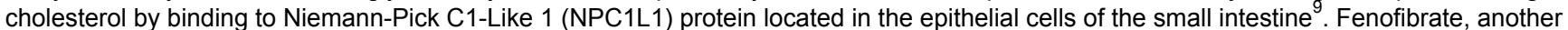
lipid-lowering drug, substantially reduces plasma concentrations of triglycerides and also moderately decreases LDL cholesterol through the peroxisome proliferator-activated receptors pathway ${ }^{10}$. Besides, omega- 3 fatty acid is reported to have the anti-atherosclerotic effect due to its ability to lower the plasma levels of $\mathrm{LDL}^{11}$.

The lipid-lowering drugs, in addition to their primary effect on lowering LDL cholesterol, have a number of beneficial pleiotropic effects including enhancing the HDL level, improving endothelial functions, reducing inflammation, and inhibiting platelet aggregations ${ }^{12,13,14}$. However, the underlying mechanism of these drugs in increasing HDL cholesterol particles and modifying their structural features are not fully understood. Since these medicines are widely prescribed to treat atherosclerosis-related cardiovascular diseases (CVDs), it is essential to further investigate their possible roles in determining morphological features and distribution of the lipid particles. The human plasma lipidome consists of approximately 600 distinct lipids and 22 different molecular types of cholesterols that are present in various sizes, shapes, densities, and compositions ${ }^{15,16,17}$. Analytical methods such as ultra-centrifugation, NMR, and gradient gel electrophoresis are used to characterize LDL and HDL particles and their subfractions ${ }^{18,19}$. However, application of these methods is limited to studies aimed at determining the effect of drugs in 
modulating morphology and assembly of the lipid particles. The flow cytometer based plaque array is a functional biochemical assay developed for detection and visualization of serum derived lipid and amyloid plaque particles ${ }^{20}$. The advantages of in vitro imaging method described in this study enable identification of lipid-modulating drug effects in altering morphology and distribution of cholesterol particles in buffer and serum samples.

\section{Preparation of Fluorescent-labeled Cholesterol and Lipid-lowering Drugs}

NOTE: Fluorescent-labeled cholesterol aggregates and statins were prepared as described in our previous article ${ }^{21}$. Please see Table of

Materials for details of reagents, imaging flow cytometer, chemistry analyzers, and data analysis software used in this study.

1. Solubilize fluorescent-labeled lyophilized cholesterol $(1 \mathrm{mg}$, Ex/Em $=495 \mathrm{~nm} / 507 \mathrm{~nm}$ ) powder in $1 \mathrm{~mL}$ of $100 \%$ alcohol.

2. Centrifuge the sample for $3 \mathrm{~min}$ at $2,040 \mathrm{xg}$ and use the supernatants containing soluble fluorescence-labeled cholesterol aggregates in the plaque array assay.

3. For the preparation of drugs, individually solubilize the powders $(2 \mathrm{mg})$ of simvastatin, lovastatin, atorvastatin, ezetimibe, and omega- 3 fatty acid in $1 \mathrm{ml}$ of $100 \%$ alcohol.

4. After centrifugation for $3 \mathrm{~min}$ at $2,040 \mathrm{xg}$, use the supernatants containing drugs in the assay.

5. Similarly, solubilize individually the powders $(2 \mathrm{mg})$ of fluvastatin, rosuvastatin, niacin, and fibrate in $1 \mathrm{~mL}$ of deionized water to make a stock solution of $2 \mathrm{mg} / \mathrm{mL}$.

6. After centrifugation for $3 \mathrm{~min}$ at $2,040 \mathrm{xg}$, use the supernatants containing drugs in the plaque array assay.

\section{Plaque Array Assay for Examining In Vitro Cholesterol Particles Formation}

1. Use chemistry analyzer-1 (see Table of Materials) to set up the plaque array assay for cholesterol particles formation. Perform the assay in a round bottom, low protein binding 96 -well plate using the reagents prepared in Section 1. Maintain the final reaction volume in each well at $200 \mu \mathrm{L}$ and perform all assays in triplicate.

1. First, load $194.5 \mu \mathrm{L}$ of phosphate buffered saline (PBS) to each well.

2. To each well, add $2.5 \mu \mathrm{L}(5 \mu \mathrm{g})$ of each lipid-lowering drug solution and shake the plate for $30 \mathrm{~s}$ by placing it on chemistry analyzer-1 reaction plate to uniformly mix the drug into solution. No drug is added to the negative control samples.

3. Finally, add $2 \mu \mathrm{L}(2 \mu \mathrm{g})$ fluorescent-labeled cholesterol aggregate solution to each well and shake the plate for $30 \mathrm{~s}$ by placing it on chemistry analyzer- 1 reaction plate.

4. Incubate the plate on a lab shaker for $2 \mathrm{~h}$ set at $37^{\circ} \mathrm{C}$ and $200 \mathrm{rpm}$.

5. After incubation, acquire the images of particles by imaging flow cytometry.

\section{Capturing Images of Cholesterol Particles Using Imaging Flow Cytometry}

1. Open the data acquisition template to load the correct instrument settings. Click "File", then select "Open Template..." and select the template file.

2. Click "Flush, Lock, Load" to prepare the instrument for sample loading.

3. When the "Load Sample" dialogue box opens, click "OK" to load $50 \mu \mathrm{L}$ of the samples from each well prepared in Section 2 into the imaging flow cytometer.

4. Click "Run|Setup" to view the particles in the imaging area in real time.

5. When the particles in the imaging area are in good focus, click "Run|Acquire" to acquire images of each object simultaneously in a highthroughput manner for dark field (side scatter/SSC), bright field (BF), green fluorescence, and yellow fluorescence.

6. Repeat steps $3.2-3.5$ to acquire $5,000-10,000$ particles from each sample.

7. Analyze all raw image files using the image analysis software for determining object fluorescence intensity and morphological variations.

1. Click the "Tools" button at the top of the image analysis software, then select "Batch Data Files" from the drop-down menu, which will open the "Batches" window.

2. In the "Batches" window, click the "Add Batch" button, which will open the "Define a Batch" window.

3. In the "Define a Batch" window, click the "Add Files" button and select the raw image files, click the "Template" button and select the appropriate data analysis template file, then select "OK" and "Submit Batches" to begin processing and analysis of the raw image files.

\section{Plaque Array Assay Based Evaluation of Lipid-lowering Drugs Effect on Purified Cholesterol Particles}

1. As described in step 2.1, perform the cholesterol particles formation assay using purified VLDL, LDL, and HDL lipoproteins/ particles. Perform the plaque array assay in a 96 -well plate.

1. First, load all wells with PBS; use a $200 \mu \mathrm{L}$ final volume for each well.

2. For negative control, add $4 \mu \mathrm{g}$ VLL, LDL, or HDL proteins/particles individually in each well without drugs and shake the plate for $30 \mathrm{~s}$. 
1. For examining the lipid-lowering drug effect, add $4 \mu \mathrm{g}$ VLDL, LDL, or HDL proteins/particles individually to each well and shake the plate for $30 \mathrm{~s}$.

2. For wells added with $4 \mu \mathrm{g}$ VLDL, LDL, or HDL, add $2.5 \mu \mathrm{L}(5 \mu \mathrm{g})$ ezetimibe, lovastatin, simvastatin, or niacin solution and shake the plate for $30 \mathrm{~s}$ by placing it in chemistry analyzer-1.

3. Finally, add $2 \mu \mathrm{L}(2 \mu \mathrm{g})$ fluorescence-labeled cholesterol aggregate solution to all wells and shake the plate for $30 \mathrm{~s}$ by placing it on chemistry analyzer- 1 reaction plate.

3. Incubate the plate for $2 \mathrm{~h}$ in a lab shaker set at $37^{\circ} \mathrm{C}$ and $200 \mathrm{rpm}$.

4. Acquire the samples using imaging flow cytometry following steps $3.1-3.5$

5. Analyze raw image files using image analysis software as described in step 3.7 .

6. In the analysis template, use the following gating scheme for identification of cholesterol particles subpopulations.

1. On a plot of green channel saturated pixel count (x-axis) and dark field channel saturated pixel count (y-axis), reject objects with one or more saturated pixels in either channel.

2. Plot the objects/particles without saturated pixels using green channel intensity (x-axis) and SSC intensity (y-axis): objects fall into one of three regions (VLDL, LDL, HDL) based on their green channel and SSC intensities.

NOTE: These gates were created based on data generated from control experiments carried out using purified VLDL, LDL, and HDL particles/proteins without the drug.

7. To determine the lipid-lowering drug effect for each sample, calculate the total lipoprotein particles concentrations, as well as the percentage of each subpopulation (VLDL, LDL, HDL) as described in step 6.5.

1. Calculate the particle concentrations (particles $/ \mathrm{mL}$ ) using the following equation:

[Particle $]=\frac{\text { particle count }}{(\text { sample flow rate })(\text { acquisition time })}$

NOTE: The VLDL, LDL, and HDL gating regions in the fluorescence dot-plot detect $\sim 80 \%$ of their respective subpopulations, and the remaining $\sim 20 \%$ of the particles overlap with other gating regions.

\section{Measurement of Lipid Concentrations in the Serum Samples}

1. For determining concentrations of LDL, HDL, cholesterol, and triglycerides by chemistry analyzer-2, use serum samples obtained from agematched normal and dyslipidemia subjects.

2. Define abnormal concentration of LDL, HDL, cholesterol, and triglycerides based on their concentration identified beyond the upper and lower reference values in serum samples.

3. Follow the normal reference value recommended by the reagent manufacturer: cholesterol (4 - $200 \mathrm{mg} / \mathrm{dL}), \mathrm{HDL}$ ( 3.0 - $65 \mathrm{mg} / \mathrm{dL}), \mathrm{LDL}$ (4 $100 \mathrm{mg} / \mathrm{dL})$, and triglycerides (9 - $200 \mathrm{mg} / \mathrm{dL})$.

4. Identify serum samples that have an abnormal value as either below the lower reference value or above the higher reference value for cholesterol and triglycerides, and use them for screening in the presence and absence of lipid-lowering drugs.

\section{Analyzing the Effect of Drugs on Cholesterol Particles Formation in Serum Samples}

1. Use the chemistry analyzer-1 to set up the plaque array assay for cholesterol particles formation in serum samples. Perform the assay in a round bottom, low protein binding 96 -well plate. Use a final reaction volume of $200 \mu \mathrm{L} /$ well and perform all assays in triplicate.

2. Prepare the plate by loading reagents in a stepwise manner.

1. Prepare the control wells.

2. Load $193 \mu \mathrm{L}$ of PBS to each well.

3. Add $2.5 \%(\mathrm{v} / \mathrm{v})$ patient serum (only one serum sample per well) and shake the plate for $30 \mathrm{~s}$ by placing it on chemistry analyzer reaction plate.

4. Add $2 \mu \mathrm{L}(2 \mu \mathrm{g})$ of fluorescence-labeled cholesterol aggregate solution into each well and shake the plate for $30 \mathrm{~s}$ by placing it on a chemistry analyzer- 1 reaction plate.

3. Prepare the wells with drugs.

1. Load $191 \mu \mathrm{L}$ of PBS to each well.

2. Add $2.5 \%(\mathrm{v} / \mathrm{v})$ patient serum (only one serum sample per well) and shake the plate for $30 \mathrm{~s}$ by placing it on a chemistry analyzer-1 reaction plate.

3. Add $2 \mu \mathrm{L}$ ezetimibe, lovastatin, simvastatin or niacin solution $(4 \mu \mathrm{g})$ to all wells except the negative control wells. Add only one drug per well and shake the plate for $30 \mathrm{~s}$ by placing it on a chemistry analyzer-1 reaction plate.

4. Add $2 \mu \mathrm{L}$ of fluorescence-labeled cholesterol aggregate solution $(2 \mu \mathrm{g})$ into each well and shake the plate for $30 \mathrm{~s}$ by placing it on chemistry analyzer- 1 reaction plate.

4. Load drugs only after all wells (control and drug-treated) have been loaded with their respective serum samples, and add fluorescencelabeled cholesterol at the end of this step.

5. Incubate the plate for $2 \mathrm{~h}$ in a lab shaker set at $37^{\circ} \mathrm{C}$ and $200 \mathrm{rpm}$.

6. Acquire the samples by imaging flow cytometer following settings described in steps $3.1-3.6$.

7. Use the image analyzing software for batch processing all the image files using the same template as described in steps 3.7 .

1. Perform the data analysis by drawing gates on the plot as described in steps 4.5 and 4.6.

8. To determine the lipid-lowering drug effect/response (low, moderate, and high) for each sample, calculate the total lipoprotein particle concentration, as well as the percentage of the total particles comprising of VLDL, LDL, and HDL subpopulations. 
9. Plot the VLDL, LDL, and HDL populations on a histogram of the object's bright field image, width subtracted from length (length - width), and gate into either globular or linear regions: objects with (length - width) $\leq 2 \mu \mathrm{m}$ fall into the globular region for calculating the percentage of globular and linear shaped particles.

10. Compare the percentage of globular and linear shaped LDL and HDL cholesterol particles identified for each serum sample incubated with and without each drug. Among triplicate values obtained for each serum sample, accept the percentage of globular and linear shaped LDL and $\mathrm{HDL}$ particles that fall within $\mathrm{SD} \pm 2$ range for determination of the drug effect.

Representative Results

\section{Plaque Array Assay Based Analysis of Lipid-lowering Drug Effects on Cholesterol Particles Formation:}

For evaluating the effect of statins in modulating morphology of cholesterol particles, fluorescent-labeled cholesterol aggregates were individually incubated with lovastatin, simvastatin, atorvastatin, rosuvastatin, and fluvastatin in the buffer. All these samples were acquired using imaging flow cytometry to capture images of the cholesterol particles for morphology analysis as shown in Figure $\mathbf{1}$ and Figure $\mathbf{2}$. Interestingly, analyzing the drug effect on cholesterol particles formation in buffer indicated that lovastatin, simvastatin, and atorvastatin induce the formation of a heterogeneous population of cholesterol particles displaying diverse sizes and shapes, Figure 3a-c. Conversely, the cholesterol particles formed in the presence of rosuvastatin, fluvastatin, and the negative control (without drug) were homogeneous in shape and morphology as shown in Figure 3d-f. In addition, it was observed that lovastatin, simvastatin, and atorvastatin induced the formation of cholesterol particles exhibiting both globular and linear strand morphologies, whereas rosuvastatin and fluvastatin induced the formation of cholesterol particles with only globular morphology. The statins effect on inducing the linear strands formation was found in the order of $16 \%$ for lovastatin, $2 \%$ for simvastatin, and $0.2 \%$ for atorvastatin.

To further evaluate the effect of lipid-lowering drugs on particles formation, fluorescent-labeled cholesterol aggregates were individually incubated with ezetimibe, fibrate, niacin, and omega-3 fatty acid. As observed with the statins, these drugs induced the formation of cholesterol particles with heterogeneous sizes and shapes as displayed in Figure 4a-d. Among them, ezetimibe induced the formation of cholesterol particles exhibiting both globular and linear strand morphologies whereas fibrate, niacin, and omega-3 fatty acid induced the formation of only globularshaped cholesterol particles. Accordingly, the effect of drugs on the linear strand shaped cholesterol particles formation was in the order of $3 \%$ for ezetimibe, $0 \%$ for fibrate, $0 \%$ for niacin, and $0 \%$ for omega- 3 fatty acid.

The morphological analysis revealed each globular or linear strand shaped cholesterol particle is composed of many smaller particles linked together. The sizes of fluorescent positive globular cholesterol particles identified are in the range of $\sim 2-30 \mu \mathrm{m}^{2}$, whereas the sizes of the linear shaped particles are in the range of $\sim 2-60 \mu \mathrm{m}^{2}$.

\section{Analyzing the Effect of Lipid-Lowering Drugs on Purified VLDL and LDL Particles:}

To further examine the cholesterol particles formation in the presence of lipoproteins, the fluorescent-labeled cholesterol aggregates were individually incubated with purified VLDL, LDL, and HDL proteins/particles. The results showed, compared to the incubation with LDL and HDL particles, that the cholesterol aggregates incubated with VLDL proteins caused the formation of a higher number of cholesterol particles, Figure 5. In addition, two major fractions of cholesterol particles were observed in VLDL populations suggesting their partial transformation into a LDL fraction during the incubation with the fluorescent-labeled cholesterol aggregates. The image analysis of particles indicated the presence of both globular $(\sim 97 \%)$ and linear shaped $(\sim 3 \%)$ particles among VLDL, LDL, and HDL populations. The size ranges of globular particles are $\sim 2-30$ $\mu \mathrm{m}^{2}$, whereas the size ranges of linear particles are $\sim 2-60 \mu \mathrm{m}^{2}$.

For examining the lipid-lowering drug effect on purified particles, the VLDL and LDL particles were individually incubated with ezetimibe, lovastatin, simvastatin, and niacin. As a result, compared to control experiments without the drug, the drug effect observed on VLDL particles formation was higher in ezetimibe, simvastatin, lovastatin, and niacin. The LDL particles incubated with the drugs showed a major single fraction and the drug-induced effect on particles formation was higher in ezetimibe, simvastatin, lovastatin, and niacin, Figure 6.

\section{Variations of the Lipid-lowering Drug Effects in Altering the Distribution of Cholesterol Particles in Serum Samples:}

The preceding experiments were performed in the buffer solution with purified lipoproteins for evaluating the drug effect. Hence, in the next step, the effectiveness of lipid-lowering drugs on cholesterol particles formation was examined using 50 serum samples collected from 25 subjects with dyslipidemia and 25 age-matched normal subjects. The drug response in each serum sample was measured based on changes in the profile of cholesterol particles formation in the presence and absence of drugs. In the plaque array assay, each serum sample was screened against ezetimibe, lovastatin, simvastatin, and niacin drugs. The results revealed disparity among these drugs in modulating the distribution of VLDL, LDL, and HDL particles in serum samples, particularly their effect on reducing LDL and increasing HDL cholesterol particles formation. Three representatives of dyslipidemia serum samples exhibiting unique responses to the lipid-lowering drugs are shown in Figure 7.

\section{Identification of the Drug Effect on Modulating Morphology of Serum Derived LDL and HDL Cholesterol Particles:}

The phenotype analysis of serum-derived cholesterol particles revealed the presence of both linear strands and globular shaped VLDL, LDL, and HDL subpopulations, thus confirming a similar morphology identified in the experiments performed both in the buffer and with purified lipoprotein particles as shown in Figure 8. However, the distribution of the globular and linear shaped cholesterol particles subpopulations widely varied among dyslipidemia and age-matched normal subjects. Notably, the control assays performed without the drugs showed differences in the distribution of linear strand shaped LDL cholesterol particles between dyslipidemia (mean of $2.0 \%$ ) and age-matched normal (mean of $1.3 \%$ ) serum samples. Similarly, an increased level of linear strand shaped HDL cholesterol particles was observed in dyslipidemia samples (mean of $18.3 \%$ ) compared to the age-matched (mean of $11.1 \%$ ) serum samples. In correlation, the assays performed in the presence of drugs in dyslipidemia serum samples showed a significant reduction in linear shaped HDL cholesterol particles formation for simvastatin (mean of $8.3 \%$ ), ezetimibe (mean of $11.5 \%$ ), lovastatin (mean of $11.7 \%$ ), and no reduction for niacin (mean of $18.3 \%$ ). In addition, a decrease in the formation of linear shaped LDL cholesterol particles was observed in dyslipidemia serum samples when incubated with the drugs displayed in Table 1. 
Furthermore, the assays performed in the presence of drugs in age-matched control serum samples showed significant reduction in the linear shaped HDL cholesterol particles in simvastatin (mean of 5.0\%), ezetimibe (mean $8.2 \%$ ), lovastatin (mean $8.7 \%$ ), and niacin (mean $10.8 \%$ ) as shown in Table 2. Both dyslipidemia and age-matched normal serum samples exhibiting drug-induced reduction in the linear shaped LDL and HDL cholesterol particles showed a relative increase in globular shaped cholesterol particles (data not shown).

(a)

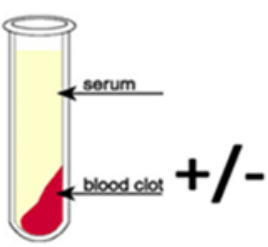

Buffer or

Serum (b)

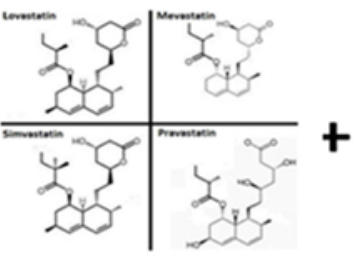

Lipid

modulator (c)

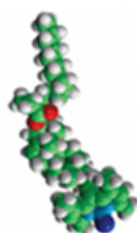

Fluorescence

cholesterol (d)

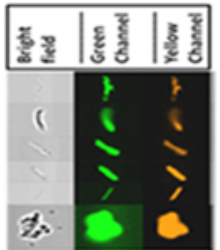

Lipid particles image library

Figure 1: Diagram illustrating the process of in vitro visualization of cholesterol particles morphology. (a, b) The addition of lipid-lowering drug in buffer or serum samples. (c) The addition of fluorescence-labeled soluble cholesterol aggregates to the samples. (d) Acquiring the resulting samples for morphological analysis of insoluble cholesterol particles using imaging flow cytometry. Scale bars $=10 \mu \mathrm{m}$. Please click here to view a larger version of this figure.

(a)

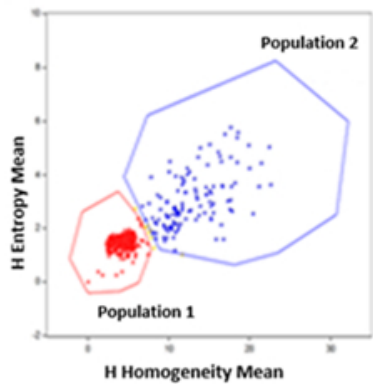

(b)

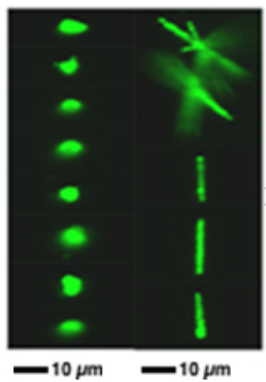

(d)

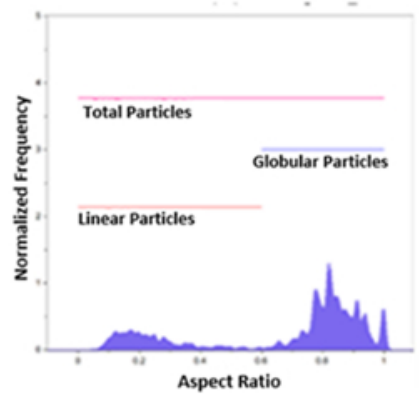

Figure 2: Identification of two distinct morphologies of cholesterol particles using imaging flow cytometry. (a) Particles are segregated into linear or globular populations based on textural analysis of the bright field images. Specifically, the dot-plot of Mean $\mathrm{H}$ Homogeneity ( $\mathrm{x}$-axis) and Mean H Entropy (y-axis) contains two gated regions for detecting globular (red) and linear strand shaped (blue) cholesterol particles. (b) Images displaying morphology of globular shaped particles identified in population 1. (c) Images of linear strand shaped particles identified in population 2. (d) Histogram displaying the distribution of all fluorescent positive cholesterol particles used to determine their concentration and subpopulations. Scale bars $=10 \mu \mathrm{m}$. Please click here to view a larger version of this figure.

(a)

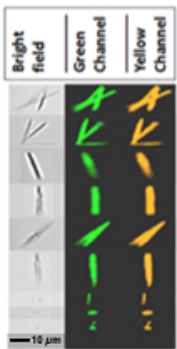

(b)

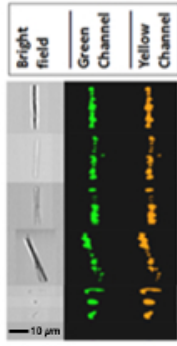

(c)

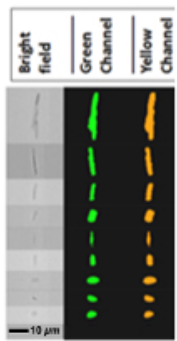

(d)

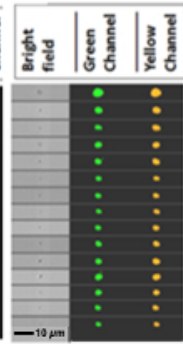

(e)

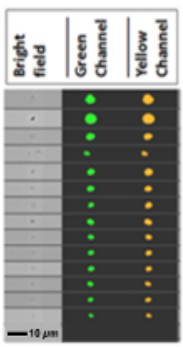

(f)

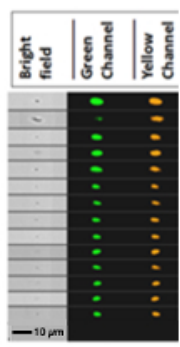

Figure 3: Image galleries displaying the effect of statins in modulating cholesterol particles formation. Bright field refers to the area similar to FSC in the conventional flow cytometer. Green channel refers to fluorescence emission detected in the $505-560 \mathrm{~nm}$, and Yellow channel refers to fluorescence emission detected in the 560-595 nm. (a-e) Image galleries displaying morphology of cholesterol particles formed in the presence of lovastatin, simvastatin, atorvastatin, rosuvastatin, and fluvastatin, respectively. (f) Cholesterol particles formation in the absence of statin (Negative control). Scale bars $=10 \mu \mathrm{m}$. Please click here to view a larger version of this figure. 
(a)

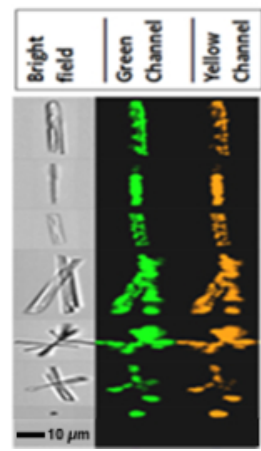

(b)

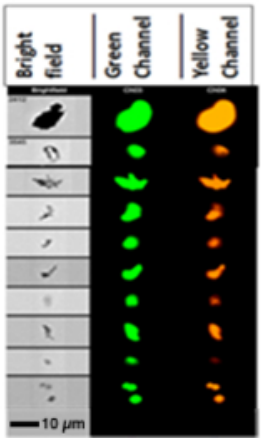

(c)

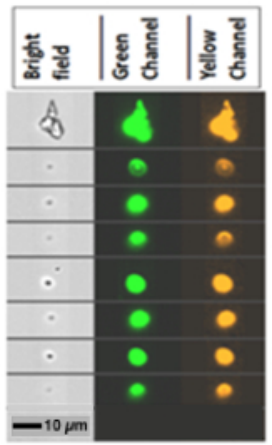

(d)

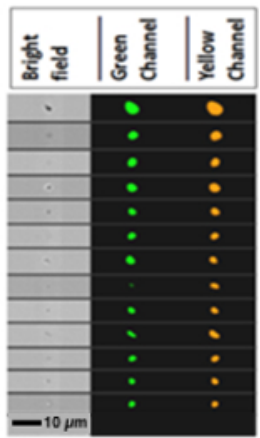

Figure 4: Demonstrating the differential effect of lipid-lowering drugs in modulating cholesterol particles formation. (a-d) Image galleries displaying morphology of cholesterol particles formed in the presence of ezetimibe, niacin, fibrate, and omega-3 fatty acid, respectively. Green channel refers to fluorescence emission detected in the $505-560 \mathrm{~nm}$, and Yellow channel refers to fluorescence emission detected in the $560-595 \mathrm{~nm}$. Scale bars $=10 \mu \mathrm{m}$. Please click here to view a larger version of this figure.

(a)

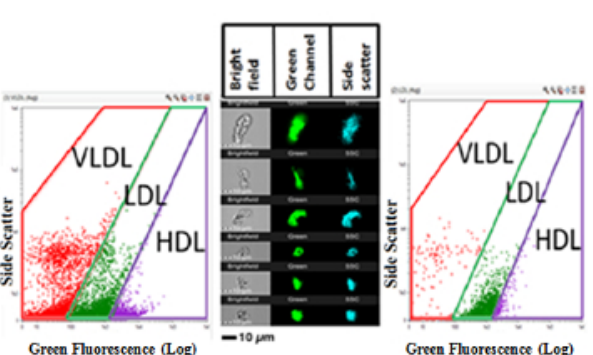

(d)

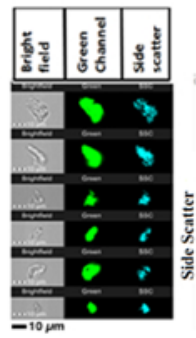

(e)

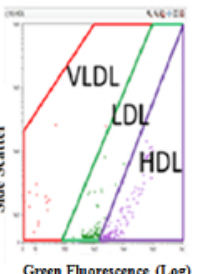

(f)

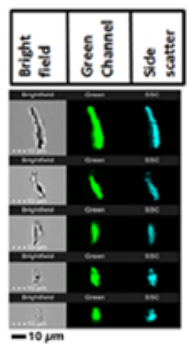

Figure 5: Analysis of VLDL, LDL, and HDL cholesterol particles formation in the absence of drug. Dot-plots: X-axis displays a spectrum of cholesterol particles detected in the green fluorescence channel $(505-560 \mathrm{~nm})$ and Y-axis displays side scatter. Gating shows regions of VLDL, LDL, and HDL particles detected in the fluorescence dot-plots. (a) Cholesterol particles formation in the presence of purified VLDL. (b) Representative images of VLDL particles. (c) Cholesterol particles formation in the presence of purified LDL. (d) Representative images of LDL particles. (e) Cholesterol particles formation in the presence of purified HDL. (f) Representative images of HDL particles. Scale bars $=10 \mu \mathrm{m}$. Please click here to view a larger version of this figure.

(a)

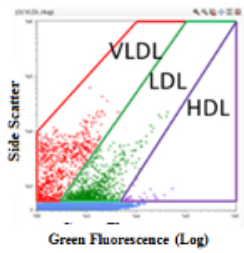

(f) (b)

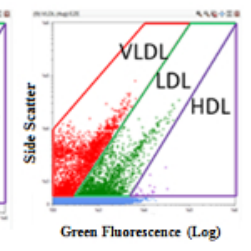

(g) (c)

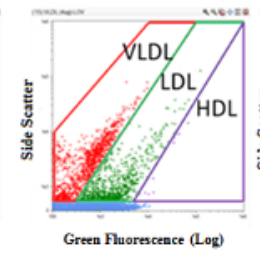

(h) (d)

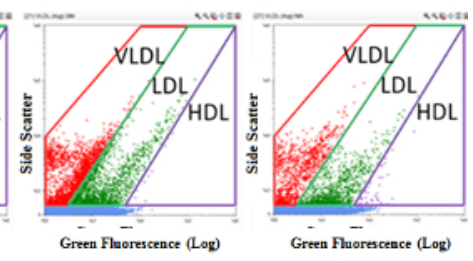

(i) (e)

(j)
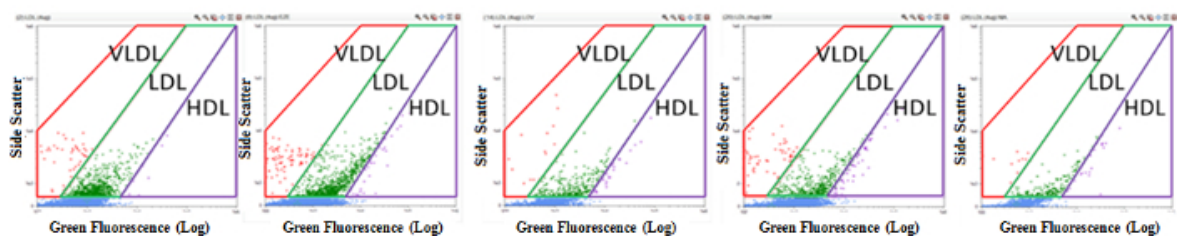

Figure 6: Demonstrating the effect of lipid lowering drugs on purified VLDL and LDL cholesterol particles. (a) VLDL particles without the drug. (b) VLDL particles incubated with ezetimibe. (c) VLDL particles incubated with lovastatin. (d) VLDL particles incubated with simvastatin. (e) VLDL particles incubated with niacin. (f) LDL particles incubated without the drug. (g) LDL particles incubated with ezetimibe. (h) LDL particles incubated with lovastatin. (i) LDL particles incubated with simvastatin. (j) LDL particles incubated with niacin. Please click here to view a larger version of this figure. 
(a)

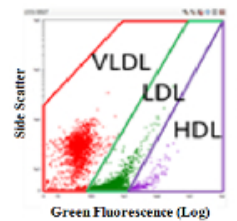

(f)

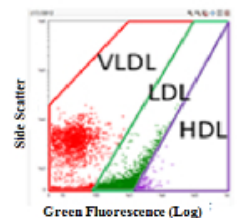

(k)

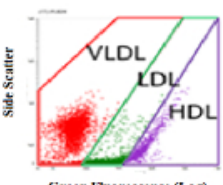

(c)

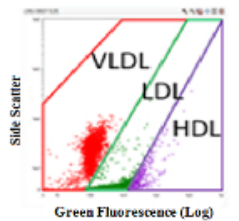

(g)

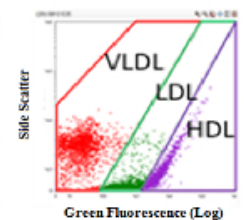

(I)

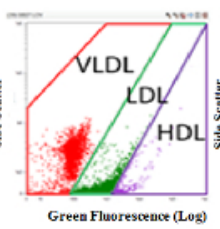

(h) (d)

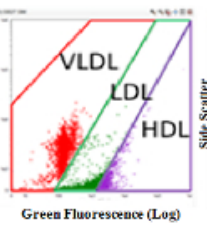

(i) (e)

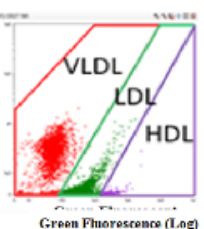

(j)

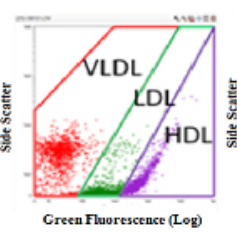

(m)

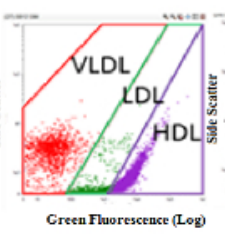

(n)

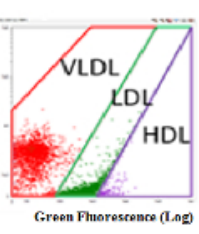

(o)

Figure 7: Fluorescence dot-plots showing a differential effect of lipid lowering drugs in modulating VLDL, LDL, and HDL cholesterol particles formation in serum samples. Top row, screening of serum 1 showing low level drug response in increasing 0 to $15 \% \mathrm{HDL}$ particles formation; middle row, screening of serum 2 showing moderate level drug response in increasing 16 to $50 \%$ HDL particles; Bottom row, screening of serum 3 showing higher level drug response in increasing 51 to $100 \%$ HDL particles. (a, f, k) Serum samples without drugs. (b, $\mathbf{g}$, I) Serum samples incubated with ezetimibe. (c, h, $\mathbf{m}$ ) Serum samples incubated with lovastatin. (d, i, $\mathbf{n}$ ) Serum samples incubated with simvastatin. $(\mathbf{e}, \mathbf{j}, \mathbf{o})$ Serum samples incubated with niacin. Please click here to view a larger version of this figure.

(a)

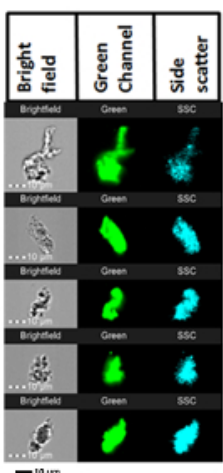

(b)

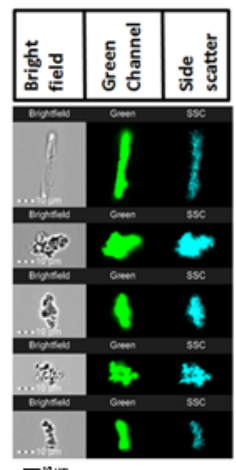

(c)

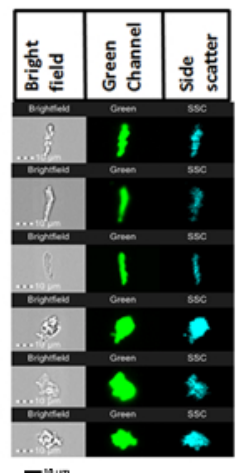

(d)

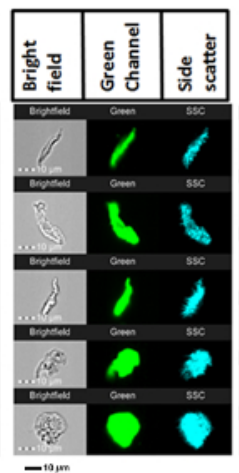

(e)

Figure 8: Image galleries displaying morphology of cholesterol particles identified in serum sample 1. Green channel shows images of the fluorescence emission from the particles; side scatter (cyan channel) shows images of the excitation laser light scattered by the particles. (a) Globular and linear shaped cholesterol particles formed without the drug. (b, c, d, e) Cholesterol particles formed in the presence of ezetimibe, lovastatin, simvastatin, and niacin, respectively. Scale bars $=10 \mu \mathrm{m}$. Please click here to view a larger version of this figure. 


\begin{tabular}{|c|c|c|c|c|c|c|c|c|c|c|}
\hline \multirow[t]{2}{*}{ Serum ID } & \multicolumn{2}{|c|}{ Without drug (Control) } & \multicolumn{2}{|c|}{ With Ezetimibe } & \multicolumn{2}{|c|}{ With Lovastatin } & \multicolumn{2}{|c|}{ With Simvastatin } & \multicolumn{2}{|c|}{ With Niacin } \\
\hline & $\begin{array}{l}\text { \% Linear, } \\
\text { LDL } \\
\text { particles }\end{array}$ & $\begin{array}{l}\text { \% Linear, } \\
\text { HDL } \\
\text { particles }\end{array}$ & $\begin{array}{l}\text { \% Linear, } \\
\text { LDL } \\
\text { particles }\end{array}$ & $\begin{array}{l}\text { \% Linear, } \\
\text { HDL } \\
\text { particles }\end{array}$ & $\begin{array}{l}\text { \% Linear, } \\
\text { LDL } \\
\text { particles }\end{array}$ & $\begin{array}{l}\text { \% Linear, } \\
\text { HDL } \\
\text { particles }\end{array}$ & $\begin{array}{l}\text { \% Linear, } \\
\text { LDL } \\
\text { particles }\end{array}$ & $\begin{array}{l}\text { \% Linear, } \\
\text { HDL } \\
\text { particles }\end{array}$ & $\begin{array}{l}\text { \% Linear, } \\
\text { LDL } \\
\text { particles }\end{array}$ & $\begin{array}{l}\text { \% Linear, } \\
\text { HDL } \\
\text { particles }\end{array}$ \\
\hline PNDS-01 & 1.73 & 45.2 & 0.81 & 18.1 & 0.61 & 16.1 & 0.59 & 13.9 & 2.26 & 33.6 \\
\hline PNDS-02 & 2.86 & 35.9 & 1.26 & 30.9 & 1.27 & 22.7 & 0.73 & 15.2 & 3.03 & 37.7 \\
\hline PNDS-03 & 2.04 & 35.8 & 0.87 & 4.82 & 1.02 & 4.14 & 0.36 & 3.06 & 0.45 & 9.57 \\
\hline PNDS-04 & 2.56 & 32.9 & 1.15 & 21.8 & 1.12 & 18.6 & 0.77 & 17.2 & 3.37 & 36.5 \\
\hline PNDS-05 & 0.42 & 29.2 & 0.24 & 5.62 & 0.22 & 8.72 & 0.16 & 9.91 & 0.35 & 22.2 \\
\hline PNDS-06 & 1.8 & 28.1 & 0.4 & 16.8 & 0.62 & 15 & 0.42 & 9.27 & 2.28 & 38.4 \\
\hline PNDS-07 & 1.8 & 26.5 & 0.85 & 10.5 & 1.18 & 19.9 & 0.62 & 7.32 & 1.29 & 23.4 \\
\hline PNDS-08 & 0.98 & 22.8 & 0.86 & 7.28 & 1.55 & 10.2 & 0.14 & 5.98 & 0.59 & 13.3 \\
\hline PNDS-09 & 3.87 & 22.1 & 1.98 & 9.56 & 1.87 & 10.3 & 1.46 & 7.96 & 2.86 & 9.88 \\
\hline PNDS-10 & 4.46 & 21.9 & 2.57 & 13.6 & 4.04 & 17.1 & 2.28 & 11.9 & 0.71 & 25.7 \\
\hline PNDS-11 & 1.57 & 19.2 & 1.15 & 9.24 & 1.37 & 6.98 & 0.74 & 5.03 & 1.37 & 16 \\
\hline PNDS-12 & 1.06 & 16.7 & 0.66 & 4.38 & 0.7 & 4.74 & 0.99 & 6.36 & 1.14 & 4.73 \\
\hline PNDS-13 & 4.85 & 16.6 & 1.28 & 30.4 & 1.4 & 32.6 & 0.8 & 16.6 & 4.02 & 31 \\
\hline PNDS-14 & 2.08 & 16 & 0.68 & 15.4 & 0.64 & 16.5 & 1.97 & 10.2 & 1.25 & 20.11 \\
\hline PNDS-15 & 1.5 & 11.9 & 1.14 & 13.3 & 1.21 & 11.4 & 0.8 & 6.12 & 1.38 & 4.59 \\
\hline PNDS-16 & 1.82 & 10.4 & 2.04 & 9.59 & 1.24 & 5.62 & 0.91 & 5.38 & 1.31 & 7.61 \\
\hline PNDS-17 & 1.05 & 10.3 & 1.02 & 4.7 & 1.78 & 15.1 & 0.93 & 7.81 & 1.27 & 13 \\
\hline PNDS-18 & 1.11 & 8.76 & 0.54 & 3.68 & 0.61 & 3.51 & 1.01 & 5.03 & 1.02 & 6.69 \\
\hline PNDS-19 & 1 & 8.52 & 0.75 & 6.67 & 0.76 & 5.86 & 0.91 & 8.36 & 1.22 & 11.9 \\
\hline PNDS-20 & 3.54 & 7.92 & 3.78 & 12 & 3.56 & 5.81 & 3.28 & 8.28 & 3.44 & 12.3 \\
\hline PNDS-21 & 1.88 & 7.69 & 2.12 & 11.4 & 1.73 & 9.54 & 1.77 & 8.34 & 2.32 & 16.7 \\
\hline PNDS-22 & 1.64 & 7.17 & 0.35 & 5.75 & 0.56 & 13.2 & 0.14 & 4.33 & 1.23 & 17.8 \\
\hline PNDS-23 & 1.54 & 6.27 & 1.25 & 7.24 & 1.02 & 6.12 & 0.73 & 3.58 & 1.42 & 6.69 \\
\hline PNDS-24 & 0.53 & 6.22 & 0.52 & 4.49 & 0.91 & 5.57 & 0.54 & 4.01 & 0.65 & 10.5 \\
\hline PNDS-25 & 2.97 & 5.1 & 1.59 & 11 & 1.88 & 9 & 1.03 & 6.54 & 2.61 & 29.1 \\
\hline
\end{tabular}

Table 1: Screening of dyslipidemia serum samples in the plaque array assay show the differential effect of lipid-lowering drugs.

Compared to controls without drug (columns 2, 3), serum samples incubated with ezetimibe (columns 4,5 ), lovastatin (columns 6,7 ), simvastatin (columns 8,9 ), and niacin (columns 10,11) showed variations on inducing linear-shaped LDL and HDL cholesterol particles formation. 


\begin{tabular}{|c|c|c|c|c|c|c|c|c|c|c|}
\hline \multirow[t]{2}{*}{ Serum ID } & \multicolumn{2}{|c|}{ Without drug } & \multicolumn{2}{|c|}{ With Ezetimibe } & \multicolumn{2}{|c|}{ With Lovastatin } & \multicolumn{2}{|c|}{ With Simvastatin } & \multicolumn{2}{|c|}{ With Niacin } \\
\hline & $\begin{array}{l}\% \text { Linear, } \\
\text { LDL } \\
\text { particles }\end{array}$ & $\begin{array}{l}\text { \% Linear, } \\
\text { HDL } \\
\text { particles }\end{array}$ & $\begin{array}{l}\text { \% Linear, } \\
\text { LDL } \\
\text { particles }\end{array}$ & $\begin{array}{l}\text { \% Linear, } \\
\text { HDL } \\
\text { particles }\end{array}$ & $\begin{array}{l}\text { \% Linear, } \\
\text { LDL } \\
\text { particles }\end{array}$ & $\begin{array}{l}\text { \% Linear, } \\
\text { HDL } \\
\text { particles }\end{array}$ & $\begin{array}{l}\% \text { Linear, } \\
\text { LDL } \\
\text { particles }\end{array}$ & $\begin{array}{l}\text { \% Linear, } \\
\text { HDL } \\
\text { particles }\end{array}$ & $\begin{array}{l}\text { \% Linear, } \\
\text { LDL } \\
\text { particles }\end{array}$ & $\begin{array}{l}\text { \% Linear } \\
\mathrm{HDL} \\
\text { particles }\end{array}$ \\
\hline PNAN-01 & 2.05 & 26.8 & 0.62 & 11.9 & 0.56 & 16.9 & 0.52 & 9.28 & 1.4 & 11.9 \\
\hline PNAN-02 & 1.35 & 26.3 & 1.68 & 21.8 & 2.59 & 23.6 & 0.79 & 6.92 & 2.16 & 29.7 \\
\hline PNAN-03 & 2.4 & 24.9 & 0.53 & 7.54 & 0.57 & 13.8 & 0.55 & 5.82 & 1.7 & 4.71 \\
\hline PNAN-04 & 1.99 & 21.5 & 1.54 & 9.45 & 1.56 & 8.25 & 0.31 & 3.74 & 2.88 & 20.8 \\
\hline PNAN-05 & 1.77 & \begin{tabular}{|l|l|}
18.8 \\
\end{tabular} & 1.49 & 6.03 & 1.65 & 5.5 & 0.54 & 4.67 & 1.2 & 8.19 \\
\hline PNAN-06 & 1.12 & 15.2 & 0.67 & 4.42 & 2.68 & 13.3 & 0.5 & 2.37 & 1.54 & 16.3 \\
\hline PNAN-07 & 1.03 & 14.4 & 0.79 & 6.83 & 1.45 & 7.91 & 0.67 & 5.36 & 1.57 & 12 \\
\hline PNAN-08 & 0.98 & 14.3 & 0.88 & 4.48 & 2 & 7.1 & 0.19 & 2.66 & 1.02 & 18.1 \\
\hline PNAN-09 & 2.85 & 14.1 & 1.95 & 12.6 & 2.34 & 12.5 & 0.7 & 6.24 & 1.84 & 18.7 \\
\hline PNAN10 & 1.01 & 10.4 & 0.8 & 5.07 & 0.51 & 5.9 & 0.87 & 6.5 & 1.63 & 10.9 \\
\hline PNAN-11 & 0.92 & 12.4 & 0.21 & 9.94 & 0.29 & 3.31 & 0.29 & 6.52 & 0.58 & 10.4 \\
\hline PNAN-12 & 0.6 & 10.5 & 0.56 & 5.78 & 1.06 & 4.74 & 0.4 & 3.32 & 0.91 & \begin{tabular}{|l|l|}
11.8 \\
\end{tabular} \\
\hline PNAN-13 & 1.25 & 10.3 & 0.45 & 3.79 & 0.67 & 6.53 & 0.27 & 3.17 & 0.8 & 6.28 \\
\hline PNAN-14 & 1.03 & 9.86 & 1.12 & 8.51 & 1.05 & 6.91 & 0.6 & 5.94 & 1.05 & 8.14 \\
\hline PNAN-15 & 2.28 & 8.1 & 1.93 & \begin{tabular}{|l|}
10.4 \\
\end{tabular} & 2.14 & 8.86 & 1.56 & 6.84 & 2.31 & 8.61 \\
\hline PNAN-16 & 1.98 & 7.69 & 0.45 & 4.36 & 1 & 5.46 & 0.27 & 2.89 & 0.49 & 4.12 \\
\hline PNAN-17 & 1.72 & 6.72 & 0.75 & 14.8 & 0.74 & 9.26 & 0.49 & 5.58 & 1.98 & 12.8 \\
\hline PNAN-18 & 2.45 & 6.38 & 0.85 & 16.8 & 0.89 & 14.2 & 0.58 & 5.9 & 1.8 & 20.6 \\
\hline PNAN-19 & 1.67 & 5.12 & 0.58 & 8.63 & 0.65 & 5.7 & 0.64 & 8.8 & 1.88 & 2.08 \\
\hline PNAN-20 & 1.17 & 4.41 & 0.85 & 7.77 & 0.91 & 6.43 & 0.69 & 5.08 & 1.21 & 6.12 \\
\hline PNAN-21 & 0.31 & 4.18 & 0.48 & 6.95 & 0.19 & 5.09 & 0.15 & 2.1 & 0.29 & 5.93 \\
\hline PNAN-22 & 0.77 & 4.02 & 1.24 & 7.41 & 0.61 & 5.02 & 0.29 & 3.49 & 0.42 & 3.98 \\
\hline PNAN-23 & 0.4 & 1.25 & 0.75 & 6.25 & 0.88 & 5.91 & 0.9 & 5.06 & 0.82 & 6.71 \\
\hline PNAN-24 & 0.45 & 1.1 & 0.63 & 2.5 & 0.55 & 5.32 & 0.9 & 4.3 & 0.71 & 3.5 \\
\hline PNAN-25 & 0.36 & 1 & 0.73 & 2.4 & 0.66 & 5.1 & 0.82 & 4 & 0.7 & 3.4 \\
\hline
\end{tabular}

Table 2: Screening of age-matched control serum samples in the plaque array assay show the differential effect of lipid-lowering drugs. Compared to controls without drug (columns 2, 3), serum samples incubated with ezetimibe (columns 4, 5), lovastatin (columns 6 , 7 ), simvastatin (columns 8,9 ), and niacin (columns 10,11) showed variations on inducing linear-shaped LDL and HDL cholesterol particles formation.

\section{Discussion}

In general, the distribution and functional properties of VLDL, LDL, and HDL cholesterol particles in the blood circulation are mainly determined by metabolic, genetic, epidemiological, cellular, and plasma factors ${ }^{22,23}$. In the present study, examining the effects of lipid-modifying drugs in the buffer revealed that highly lipophilic drugs such as ezetimibe, lovastatin, simvastatin, and atorvastatin induced a higher level complexity on the morphology of cholesterol particles compared to the lower level effect observed with highly hydrophilic rosuvastatin and fluvastatin drugs. These results are in good agreement with our previous study describing a non-enzymatic mechanism based effect of statins in modulating LDL and HDL cholesterol particles formation in the buffer and serum samples ${ }^{21}$. Accordingly, the results from present study revealed a non-enzymatic mechanism of action of ezetimibe, niacin, fibrate, and omega-3 fatty acid drugs that may play a direct role in modulating cholesterol particles formation. It is possible that the interactions between drugs and cholesterol aggregates leads to the assembly of large size cholesterol particles that are $2-60 \mu \mathrm{m}^{2}$, exhibiting globular and linear strand morphologies.

Besides, the results obtained using purified lipoprotein particles suggest interactions between cholesterol aggregates and plasma factors including VLDL, LDL, and HDL proteins that may alter the compositions and morphological properties of the cholesterol particles. The drug treatment results involving purified lipoprotein particles indicated a higher level drug effect on VLDL particles formation compared to their effect observed on LDL cholesterol particles formation. The lovastatin, simvastatin, and ezetimibe drugs were used as pro-drugs and their doses in the assays may be higher than the physiological concentrations.

Interestingly, screening of serum samples showed variations of drug effect on altering the profiles of VLDL, LDL, and HDL cholesterol particles formation, particularly their effect on the formations of linear shaped LDL and HDL particles. These drugs induced reduction on linear shaped 
LDL and HDL cholesterol particles formation in both dyslipidemia and age-matched normal serum samples. The drug effects observed on reducing linear shaped particles formation was higher in simvastatin, ezetimibe, lovastatin, and niacin. The identification of cholesterol particles with globular and linear strand morphologies in the normal and dyslipidemia serum samples suggests that particles with similar morphologies may form in in vivo conditions. Previous studies have identified the presence of disc and needle-like cholesterol crystals in the atherosclerotic

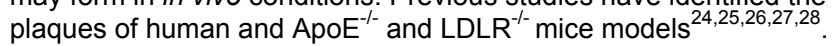

The HDL particles circulating in the blood exist as a heterogeneous mixture and the level of small and large size HDL particles along with functional activity are important factors to exert their cardio-protective effect via the reverse cholesterol transport pathway ${ }^{29,30}$. Recent studies have highlighted the importance of identifying HDL cholesterol particle subfractions for elucidating their role in multiple biological functions such as cholesterol efflux, anti-inflammation, anti-thrombotic, and anti-oxidative ${ }^{31}$. In addition, a number of studies have reported the effect of lipidlowering therapy in increasing a low to moderate level of HDL in the plasma ${ }^{1,5,21}$. Accordingly, the results from this study provide new insights on morphological features of cholesterol particles. Notably, the detection of a higher level of linear shaped HDL cholesterol particles in the serum samples of dyslipidemia subjects suggests that they may be the reliable biomarker for both diagnosis and evaluating effects of lipid-modifying drugs in patients. However, further investigation is required using large clinical samples to better understand the cholesterol particles with distinct morphologies and their association to CVD.

In the plaque array assay for examining the drug effect on assembly of cholesterol particles, we used $2 \mu \mathrm{g}$ of fluorescence labeled cholesterol aggregates and $5 \mu$ gof each drug because: (1) drugs competitively bind to both fluorescence labeled cholesterol and endogenous lipids present in the serum samples; (2) from each sample, we acquired 5,000 to 10,000 cholesterol particles that are assembled into large sizes and shapes ranging from $\sim 2-60 \mu^{2} ;(3)$ we observed a wide variations of drug response among serum samples incubated with the drugs (doses 300 ng to $5 \mu \mathrm{g}$ ) and $\sim 1-5 \%$ of them incubated with high doses showed no detectable changes in the profile of cholesterol particles formation; and (4) the interaction between cholesterol aggregates and lipid-lowering drugs is mediated by a non-enzymatic process. Hence, the concentrations of the reagents used in the assay may be higher than their physiological level.

In conclusion, we have successfully demonstrated the advantages of an in vitro imaging method described in this study for determining the effect of a broad spectrum of lipid-lowering drugs on modulating morphology and composition of cholesterol particles. The approach of visualizing and quantifying the morphology of lipid particles by employing a constellation of image analysis algorithms may help both the diagnosis of atherosclerosis and to evaluate outcomes of lipid-lowering therapy in patients.

\section{Disclosures}

Dr. Madasamy received grant support from Plaxgen, Inc and has a competing financial interest. The other authors have no competing financial interests to disclose.

\section{Acknowledgements}

This work was funded by a Plaxgen Research grant awarded to SM (PLX-1008). We thank Palo Alto Medical Research Foundation Research Institute for collecting serum samples from atherosclerosis subjects under IRB approval.

\section{References}

1. Pahan, K. Lipid-lowering drugs. Cell Mol Life Sci. 63, 1165-1178 (2006).

2. Laforest, L. et al. Prevalence of low high-density lipoprotein cholesterol and hypertriglyceridaemia in patients treated with hypolipidaemic drugs. Arch Cardiovasc Dis. 102, 43-50 (2009).

3. Roberts, W.C. Preventing and arresting coronary atherosclerosis. Am Heart J. 130, 580-600 (1995).

4. Goldstein, J.L, Brown M.S. A century of cholesterol and coronaries: from plaques to genes to statins. Cell. 16, 161-72 (2015).

5. Drexel, H. Statins, fibrates, nicotinic acid, cholesterol absorption inhibitors, anion-exchange resins, omega-3 fatty acids: which drugs for which patients?. Fundam Clin Pharmacol. 23, 687-92 (2009).

6. Camelia, S and Anca, S. Statins: Mechanism of action and effects. J.Cell.Mol.Med. 5, 378-387 (2001).

7. Schaefer, E.J. et al. Comparisons of effects of statins (atorvastatin, fluvastatin, lovastatin, pravastatin, and simvastatin) on fasting and postprandial lipoproteins in patients with coronary heart disease versus control subjects. Am J Cardiol. 93,31-39 (2004).

8. Meyers, C.D., Kamanna, V.S., Kashyap, M.L. Niacin therapy in atherosclerosis. Curr Opin in Lipid. 15, $659-665$ (2004).

9. Phan, B.A., Dayspringm, T.D., Toth, P.P. Ezetimibe therapy: mechanism of action and clinical update. Vasc Health Risk Mana. 8, 415-427 (2012).

10. Leitersdorf, E. and Fruchart, J.C. Mechanism of action of fibrates on lipid and lipoprotein metabolism. Circulation. 98, 2088-2093 (1998).

11. Backes, J., Anzalone, D., Hilleman, D., Catini, J. The clinical relevance of omega-3 fatty acids in the management of hypertriglyceridemia. Lipids Health Dis. 15,118 (2016).

12. Davidson, M.H. Clinical Significance of Statin Pleiotropic Effects Hypotheses Versus Evidence. Circulation. 111, 2280-1 (2005).

13. Chinetti-Gbaguidi, G., Fruchart, J.C., Staels, B. Pleiotropic effects of fibrates. Curr Atheroscler Rep. 7, 396-401 (2005).

14. McTaggart, F., Jones, P. Effects of Statins on High-Density Lipoproteins: A Potential Contribution to Cardiovascular Benefit Effects of Statins on High-Density Lipoproteins: A Potential Contribution to Cardiovascular Benefit. CardiovascDrugs Ther. 22, 321 - 338 (2008).

15. Quehenberger, O. et al. The Human Plasma Lipidome. N Engl J Med. 365, 1812-1823 (2011).

16. Lund-Katz, S. et al. Mechanisms responsible for the compositional heterogeneity of nascent high density lipoprotein. J. Biol Chem. 288, 23150-23160 (2013).

17. Krauss, R.M. Lipoprotein subfractions and cardiovascular disease risk. Curr Opin Lipidol. 21, 305-311 (2010).

18. Krauss, R.M., and Burke, D.J. Identification of multiple subclasses of plasma low density lipoproteins in normal humans. J Lipid Res. 23, 97-104 (1982). 
19. Rosenson, R.S., et al. HDL measures, particle heterogeneity, proposed nomenclature, and relation to atherosclerotic cardiovascular Events. Clinic Chemi. 57, 392-410 (2011).

20. Madasamy, S. et al. Plaque array method and proteomics-based identification of biomarkers from Alzheimer's disease serum. Clin Chim Acta. 441, 79-85 (2015).

21. Madasamy, S. et al. Non-enzymatic mechanism of statins in modulating cholesterol particles formation. Am J Cardiol. 118, 1187- 1191 (2016).

22. Peter, O.K. Clinical relevance of the biochemical, metabolic, and genetic factors that influence low-density lipoprotein heterogeneity. Am $J$ Cardiol. 90, 30-47 (2002).

23. Weissglas-Volkov, D and Pajukanta, P. Genetic causes of high and low serum HDL-cholesterol. J Lipid Res. 51, 2032-2057 (2010).

24. Abela, G.S. Effect of statins on cholesterol crystallization and atherosclerotic plaque stabilization. Am J Cardiol. 107, 1710-1717 (2011).

25. Nidorf, S.M., Eikelboom, J.W., Thompson, P.L. Targeting cholesterol crystal-induced inflammation for the secondary prevention of cardiovascular disease. Cardiovasc Pharmacol Ther. 19, 45-52 (2014).

26. Thacker, S.G., Zarzour, A., Chen ,Y, et al. High density lipoprotein reduces inflammation from cholesterol crystals by inhibiting inflammasome activation. Immunol. 149, 306-319 (2016).

27. Kim, S.H., Lee, E.S., Lee, J.Y., et al. Multiplex coherent anti-stokes Raman Spectroscopy images intact atheromatous lesions and concomitantly identifies distinct chemical profiles of atherosclerotic lipids. Circ Res. 106, 1332-1341 (2010).

28. Lim, R.S., Suhalim, J.L., Miyazaki-Anzai, S, et al. Identification of cholesterol crystals in plaques of atherosclerotic mice using hyperspectral CARS imaging. J Lipid Res. 52, 2177-2186 (2011).

29. Rothblat, G.H., Phillips, M.C. High-density lipoprotein heterogeneity and function in reverse cholesterol transport. Curr Opin Lipidol. 21, 229-238 (2011).

30. Kontush, A. HDL particle number and size as predictors of cardiovascular disease. Front in Pharmacol. (2015).

31. Karathanasis, S.K., Freeman, L.A., Gordon, S.M., Remaley, A.T. The changing face of HDL and the best way to measure it. Clin. Chem. 63,196-210 (2017). 